\title{
Fehlervermeidung in der Kommissionierung
}

\author{
Error Prevention in Manual Order Picking
}

\author{
Tobias Rammelmeier \\ Stefan Galka \\ Willibald A. Günthner \\ Lehrstuhl für Fördertechnik Materialfluss Logistik \\ Technische Universität München
}

$\mathbf{M}$ anuelle Kommissioniersysteme sind aufgrund ihrer Flexibilität weit verbreitet, jedoch treten bei diesen auch vermehrt Kommissionierfehler auf, die weitreichende Folgen haben können. Deshalb werden zu Beginn des Beitrags die Auswirkungen und Ursachen von Kommissionierfehlern betrachtet. Der Fokus des Beitrags liegt auf der Darlegung von Möglichkeiten zur Fehlervermeidung. Dabei wird ein ganzheitlicher Ansatz verfolgt, der Maßnahmen aus den Handlungsfeldern Technik, Prozess, Organisation und Motivation umfasst. Als Ausblick auf zukünftige technische Hilfsmittel zur Fehlervermeidung wird das Augmented-Reality-unterstützte System Pick-by-Vision aus der Forschung vorgestellt, das wesentliche Vorteile existierender Techniken der Informationsbereitstellung vereinigt.

[Schlüsselwörter: Kommissionierung, Kommissionierfehler, Fehlervermeidung, Augmented Reality, Pick-by-Vision]

D ue to their high flexibility manual order picking systems are widely-used. However, in these picking systems an increased number of picking errors occurs, which can have far-reaching consequences. Therefore at the beginning of the contribution impact and causes of picking errors are examined. The contribution focuses on measures for the avoidance of picking errors. In doing so, a holistic approach is pursued, which includes measures concerning the following activity fields: technology, process, organization and motivation. As an outlook on future technical devices for the prevention of picking errors, research activities concerning the augmented reality based picking system Pick-by-Vision, which combines the major advantages of existing technologies for the provision of information, are presented.

[Keywords: order picking, picking error, error prevention, augmented reality, Pick-by-Vision]

\section{Einleitung}

Die Kommissionierung ist die zentrale Funktion der Lagerlogistik. Ihre hohe Bedeutung zeigt sich auch in den Kosten, die diese verursacht. Von den Logistikkosten, die abhängig von der Branche 5 bis $9 \%$ der Gesamtkosten [Str08] betragen, entfallen 10 bis $50 \%$ auf die Lagerkosten und davon wiederum etwa $50 \%$ auf die Kommissionierung [Fra01].

Hinsichtlich des Automatisierungsgrades von Kommissioniersystemen existieren unterschiedliche Realisierungsformen. Zwischen vollständig automatisierten Lösungen und manuellen Kommissioniersystemen existieren viele Zwischenstufen, bei denen beispielsweise die Bereitstellung der Artikel dynamisch erfolgt, und die Entnahme manuell durchgeführt wird. Die häufigste in der Praxis anzutreffende Art der Kommissionierung ist die manuelle Kommissionierung [Str05]. Oft wird dabei nach dem Prinzip Person-zur-Ware (PzW) gearbeitet. Die Ware wird bei dieser Art der Kommissionierung statisch, meist in Regalen, bereitgestellt. Die relevanten Informationen erhält der Kommissionierer häufig in Form einer Papierliste. Ein wesentlicher Vorteil neben den geringen Investitionen der manuellen PzW-Kommissionierung ist deren hohe Flexibilität. Durch seine kognitiven Fähigkeiten und das flexible Tast- und Greifvermögen [Arn03] ist der Mensch als Kommissionierer auch in Lägern mit inhomogenem oder ständig wechselndem Sortiment einsetzbar. Nachteilig ist die - verglichen mit Kommissionierautomaten - geringe Kommissionierleistung und die Tatsache, dass Menschen Fehler machen. Im Rahmen einer Untersuchung des Lehrstuhls für Fördertechnik Materialfluss Logistik (fml) der Technischen Universität München zeigte sich, dass bei der klassischen Belegkommissionierung eine Fehlerquote von durchschnittlich ca. 0,3\% auftritt.

Die Folgen von Kommissionierfehlern sind vielfältig. Je später Fehler entdeckt werden, desto schwerer wiegen ihre Auswirkungen. Werden Kommissionierfehler erst vom Kunden entdeckt, reichen die Folgen von einer teuren Nachlieferung per Express bis hin zum Kundenverlust. In vielen Unternehmen wird deshalb eine aufwendige Warenausgangskontrolle durchgeführt, die verhindern 
soll, dass eine fehlerhafte Sendung zum Kunden gelangt. Da in jedem Fall hohe Kosten entstehen, kann es sich lohnen, aktiv der Entstehung von Kommissionierfehlern vorzubeugen. Der Lehrstuhl fml der Technischen Universität München befasst sich mit der Erarbeitung von Maßnahmen zur Vermeidung von Kommissionierfehlern. Neben präventiven Maßnahmen gehört dazu auch die Entdeckung und Behebung von Fehlern unmittelbar am Ort ihrer Entstehung. Im Forschungsprojekt „Vermeidung von Kommissionierfehlern mit Pick-by-Vision“ wird hierzu eine technische Lösung auf Basis der Augmented-RealityTechnologie entwickelt, die eine Annäherung an die NullFehler-Kommissionierung ermöglichen soll.

\section{AUSWIRKUNGEN VON KOMMISSIONIERFEHLERN}

Für Unternehmen ist es erforderlich, die Logistikprozesse konsequent an den Anforderungen der Kunden auszurichten. Dies gilt sowohl für produzierende Unternehmen als auch für Logistikdienstleister [Bau08]. Die Kommissionierung als wesentlicher Logistikprozess hat dabei maßgeblichen Einfluss auf die Kundenzufriedenheit. Allgemein sind die Auswirkungen von Kommissionierfehlern abhängig vom Zeitpunkt ihrer Entdeckung. Dies soll im Folgenden anhand der Erfahrungen mehrerer befragter Industrie- und Handelsunternehmen verdeutlicht werden.

Fehler, die noch während des Kommissioniervorgangs vom Kommissionierer selbst entdeckt werden, sind zwar nicht kundenrelevant, verursachen aber dennoch Aufwand in Form von Zeit und Kosten für deren Behebung. Dieser Aufwand steigt deutlich an, wenn Fehler erst im Rahmen einer internen Kontrolle, z. B. bei der Warenausgangskontrolle, entdeckt werden. Zur Fehlerbehebung ist es in diesem Fall neben der ggf. notwendigen Wiedereinlagerung der falschen Artikel erforderlich, einen neuen Kommissionierauftrag zu generieren, um die richtigen Artikel zu kommissionieren.

Verlassen fehlerhafte Bestellungen das Unternehmen, und werden diese vom Kunden entdeckt, führt dies zur Reklamation und dem damit verbundenen Aufwand der Reklamationsabwicklung. Kommt es häufiger zu Fehlern, so kann dies auch zum Kunden- und Imageverlust führen. Gerade bei der Just-In-Time-Anlieferung in Produktions- betrieben können die Kosten für eine Nachlieferung via Express oder Schadensersatzzahlungen für einen Produktionsausfall sehr hoch sein. Unentdeckte Fehler können schwerwiegende Folgen nach sich ziehen. Ausgehend von einem falschen Bauteil, das in einem Fahrzeug oder einer Maschine eingebaut wird, können Maschinen zerstört oder sogar die Gesundheit und das Leben von Menschen gefährdet werden.

Zusammenfassend lässt sich feststellen, dass Kommissionierfehler hohe Kosten verursachen und das Kunden-Lieferanten-Verhältnis stark belasten können. Deshalb ist es empfehlenswert, Maßnahmen zu treffen, um Kommissionierfehlern präventiv entgegenzuwirken und im Falle eines Fehlers diesen möglichst frühzeitig zu erkennen und zu beheben.

\section{ARTEN Und URSACHEN VON KommissioniER- FEHLERN}

Für die zielgerichtete Auswahl von Maßnahmen zur Fehlervermeidung ist es erforderlich, die unterschiedlichen Arten und Ursachen von Kommissionierfehlern zu berücksichtigen. Es wird zwischen den folgenden Fehlerarten unterschieden [Gün09; Lol03; Dul05]:

- Typfehler: Ein falscher Artikel ist als Ersatz oder zusätzlich zum richtigen Artikel vorhanden.

- Mengenfehler: Die Stückzahl des richtigen Artikels ist zu hoch oder zu gering.

- Auslassungsfehler: Eine Position wurde übersprungen. Der Auslassungsfehler wird gelegentlich als Spezialfall des Mengenfehlers mit der Stückzahl null interpretiert.

- Zustandsfehler: Eine falsche Tätigkeit wurde am Artikel verrichtet. Häufig auftretende Beispiele sind beschädigte oder falsch etikettierte Artikel.

Im Rahmen der Untersuchung von zehn Kommissioniersystemen konnten wesentliche Ursachen von Kommissionierfehlern identifiziert und der jeweiligen Fehlerart zugeordnet werden. Abbildung 1 zeigt beispielhaft wesentliche Ursachen von Mengenfehlern. 


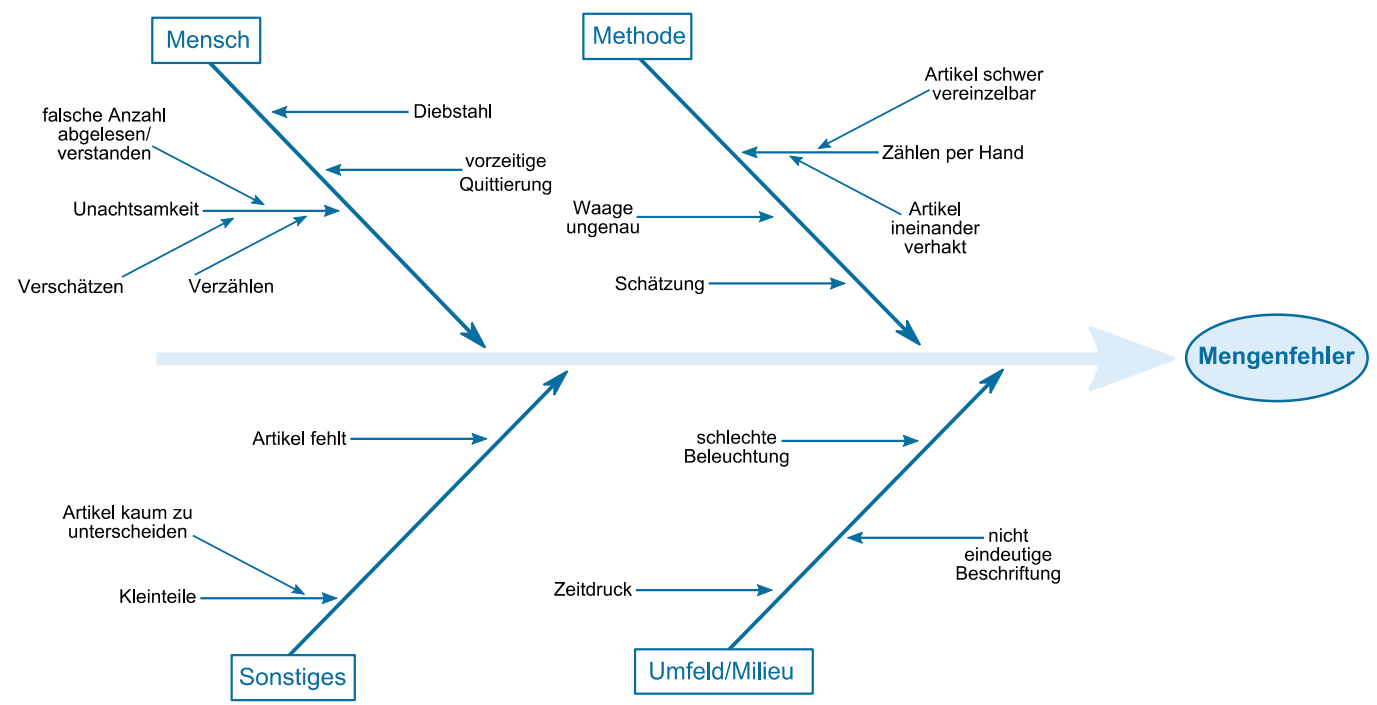

Abbildung 1. Ursache-Wirkungs-Diagramm zur Entstehung von Mengenfehlern

Die ermittelten Ursachen können den übergeordneten Kriterien „Mensch“, „Methode“, „Umfeld/ Milieu“ und „Sonstiges“ zugeordnet werden. Während unter dem Kriterium „Mensch“ die Ursachen zusammengefasst werden, die auf Eigenschaften des Menschen zurückzuführen sind, umfasst die „Methode“ Fehlerursachen, die sich durch die Gestaltung des Prozesses und die verwendeten Hilfsmittel ergeben. Das „Umfeld“ ist geprägt durch Umweltbedingungen und Faktoren wie der Gestaltung des Lagers. „Sonstiges“ umfasst alle Aspekte, die sich nicht den anderen drei übergeordneten Kriterien zuordnen lassen.

Von vielen Logistikverantwortlichen und Kommissionierern wird der Mensch als wesentliche Fehlerquelle gesehen. Unachtsamkeit führt oftmals dazu, dass sich der Kommissionierer in Abhängigkeit von der angewandten Methode verschätzt oder verzählt bzw. sich bereits bei der Informationsaufnahme eine falsche Zahl einprägt. Unterstützt wird dieser Effekt durch den meist hohen Zeitdruck.

Neben der Unachtsamkeit führt auch der falsche Umgang mit dem Kommissioniersystem zu Fehlern. Unabhängig vom eingesetzten Kommissioniersystem entstehen oftmals Fehler, wenn der Kommissionierer einen oder mehrere Prozessschritte bereits quittiert, bevor er diese ausgeführt hat.

Besonders im Bereich von unverpackten Einzelteilen treten Fehler häufig dann auf, wenn diese sehr klein sind oder sich bedingt durch ihre Form leicht verhaken. Zwar kann dem Problem schwer vereinzelbarer Artikel entgegengewirkt werden, indem die Stückzahl durch Wiegen statt durch Zählen per Hand ermittelt wird, jedoch gaben mehrere der befragten Unternehmen an, dass durch große Fertigungstoleranzen der Artikel und ungeeignete Waagen auch bei dieser Methode Mengenfehler auftreten können.

\section{MAßNAHMEN ZUR FeHLERVERMEIDUNG}

Menschen machen Fehler, demnach führt ein hoher Automatisierungsgrad zu weniger Fehlern. Diese zugegebenerweise drastische Aussage stimmt nur sehr bedingt. Auch bei automatisierten Systemen spielt der Mensch eine wesentliche Rolle. Werden z. B. Artikel falsch eingelagert, weil ein Mitarbeiter das falsche Produktlabel an einem Behälter angebracht hat, können auch in automatisierten Systemen Fehler entstehen. Nicht immer ist die Entscheidung, den Automatisierungsgrad in der Kommissionierung zu erhöhen, die richtige Wahl, da im Hinblick auf die langfristige Perspektive die Flexibilität hinsichtlich der veränderlichen Marktanforderungen fehlt. Trotz des Trends zu einem höheren Automatisierungsgrad wird der Mensch weiterhin eine wesentliche Rolle in der Kommissionierung einnehmen [Pia11]. Die hier diskutierten Ansätze konzentrieren sich auf die Fehlervermeidung in manuellen Kommissioniersystemen.

Grundsätzlich gibt es vier Handlungsfelder: technische und organisatorische Maßnahmen, eine fehlerreduzierende Prozessgestaltung und die Sensibilisierung der Mitarbeiter (Motivation), um Fehler in Kommissioniersystemen zu reduzieren. Abbildung 2 verknüpft die vier Handlungsfelder mit dem typischen Ablauf der Kommissionierung. Diese vier Handlungsfelder können nicht autark voneinander betrachtet werden. Vielmehr ist ein ganzheitlicher Ansatz zu finden, in dem die einzelnen Handlungsfelder aufeinander abgestimmt sind und sich in ihrer Wirkung ergänzen. Im Folgenden werden einzelne Aspekte für eine fehlerreduzierende Systemgestaltung diskutiert. 


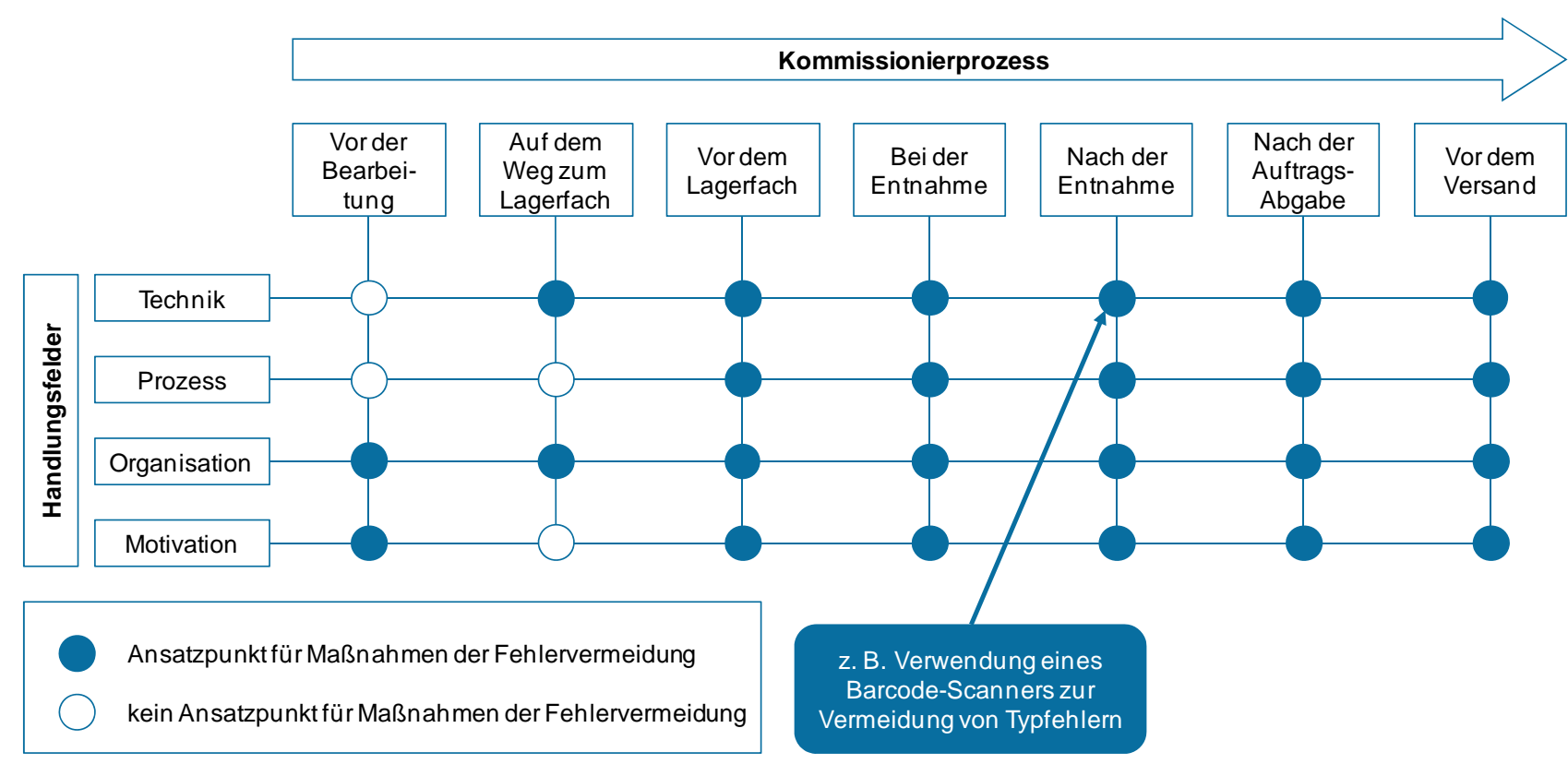

Abbildung 2. Handlungsfelder der Fehlervermeidung bei Kommissionierprozessen

Die organisatorische Gestaltung beeinflusst die Wahrscheinlichkeit, dass Kommissionierfehler entstehen können. Ein Aspekt dabei ist die Lagerplatzzuordnung für die Artikel. Aus Effizienzgründen ist eine Einlagerstrategie unter Berücksichtigung der Zugriffshäufigkeit sinnvoll. Um Fehler zu vermeiden, ist diese Strategie mit einer weiteren Maßnahme zu kombinieren. Diese sieht vor, dass ähnlich aussehende Artikel nicht in direkter Nähe eingelagert werden dürfen. Beispielsweise ist es schwieriger, zwei CDs voneinander zu unterscheiden als eine CD und ein T-Shirt. Die Trennung von ähnlichen Artikeln ist vor allem dann wichtig, wenn der Mitarbeiter die Artikelbezeichnung kennt.

Soll das volle Optimierungspotenzial zur Leistungssteigerung bei der Lagerplatzvergabe realisiert werden, ist es notwendig, dem Mitarbeiter nur noch einen codierten Lagerplatz mitzuteilen. Die Codierung ermöglicht eine eindeutige Zuordnung zwischen einem Lagerplatz und einem Artikel. Der Mitarbeiter muss die Artikel entnehmen, die sich auf dem Lagerplatz befinden. Er weiß also nicht, ob es sich um eine CD oder ein T-Shirt handelt. Dieses Vorgehen hat den Nachteil, dass Fehler bei der Einlagerung nur durch einen weiteren Abgleich erkannt werden, z. B. die Kontrolle der Artikelnummer. In der Praxis hat sich gezeigt, dass die Art der Codierung Einfluss auf die Fehlerquote hat. Wichtig ist eine Mischung aus Zahlen und Buchstaben (z. B. 2-A-4), so dass das Lagerfach einfach gefunden werden kann (Regal 2, Fach A, Spalte 4) und es nicht zu Verwechselungen kommen kann. Ein weiterer Ansatz, um Verwechselungen auszuschließen, ist die Verwendung von Begriffen aus dem Alltag. So kann ein Artikel auch im Regal „München“ in der Spalte „Odeonsplatz“ im Fach mit der Hausnummer „2“ liegen. Werden diese Bezeichnungen noch gemeinsam mit den Mitarbei- tern erarbeitet, so ist eine hohe Akzeptanz und Motivation sichergestellt.

Auch durch eine gezielte Prozessgestaltung lässt sich die Fehlerquote reduzieren. Durch die Prozessgestaltung lässt sich primär der Zeitpunkt beeinflussen, zu dem ein Fehler erkannt wird. Je früher ein Fehler erkannt wird, desto geringer ist der Aufwand für dessen Behebung. Im Prozess kann an verschiedenen Stellen eine Kontrolle vorgesehen werden. Entsprechend dem in Abbildung 2 dargestellten Ablauf kann an folgenden Stellen im Prozess eine Überprüfung stattfinden:

- beim Erreichen des richtigen Lagerfachs (Typfehler)

- bei der eigentlichen Entnahme sowie durch die Kontrolle der Anzahl der Entnahmevorgänge aus dem richtigen Lagerfach (Typfehler, Mengenfehler - wenn pro Pick eine definierte Anzahl entnommen wird)

- $\quad$ nach der Entnahme, z. B. durch Scannen des Produktbarcodes (Typ- und Mengenfehler)

- $\quad$ nach dem Abschluss des Auftrags durch den Kommissionierer oder eine weitere Person

- $\quad$ vor dem Versand

Die beiden zuletzt angesprochenen Punkte können für jeden Auftrag entweder in Form einer 100 \%-Kontrolle oder nur für ausgewählte Aufträge (Stichproben) durchgeführt werden. Allerdings wirkt diese Art der Fehlervermeidung reaktiv, d. h. bei der Entdeckung eines Fehlers ist ein weiterer Kommissioniervorgang notwendig, um diesen zu beheben. Bei den ersten drei der genannten Kontrollpunkte wird hingegen bereits ein drohen- 
der Fehler verhindert bzw. wird der Fehler am Entstehungsort entdeckt und kann schnell behoben werden.

An verschiedenen Stellen im Kommissionierprozess können zudem technische Hilfsmittel zur Fehlervermeidung eingesetzt werden. Bei einer Untersuchung von Kommissionierprozessen in der industriellen Praxis wiesen Kommissioniersysteme besonders dann eine niedrige Fehlerquote auf, wenn technische Hilfsmittel hinsichtlich der folgenden Aspekte während der Kommissionierung unterstützen:

- $\quad$ intuitive Informationsbereitstellung

- $\quad$ schrittweise Auftragsabarbeitung durch Quittierung

\section{- $\quad$ Kontrolle von Prozessschritten}

Für die schnelle und fehlerfreie Informationsaufnahme ist die intuitive Bereitstellung der relevanten Informationen von zentraler Bedeutung. Bekannte technische Lösungen hierfür sind Pick-by-Light, Pick-by-Voice oder mobile Datenterminals. Alle genannten Systeme erheben den Anspruch, aus der Vielzahl der alphanumerischen Informationen einer teilweise unübersichtlichen Pickliste nur die tatsächlich für die Bearbeitung einer Position benötigte Information bereitzustellen, indem sie den optischen oder den akustischen Sinneskanal ansprechen. Insbesondere die optische Wegführung wirkt bereits präventiv gegen einen Fehlgriff in das falsche Lagerfach. Da der Mensch über 80 \% der Information über den optischen Sinneskanal aufnimmt [Wei04], ist davon auszugehen, dass die visuelle Informationsdarstellung eine einfache und fehlerfreie Informationsaufnahme unterstützt. Die Informationsaufnahme wird dadurch unterstützt, dass die Anweisungen permanent sichtbar sind, während z. B. die Sprachanweisungen bei Pick-by-Voice nur kurz zur Verfügung stehen.

Unabhängig davon, ob die Quittierung eines Prozessschrittes, z. B. das Abschließen einer Position oder einer Entnahme, per Spracheingabe oder durch das Betätigen eines Tasters erfolgt, reduziert jeder zusätzliche Interaktionsschritt die Gefahr, eine Position zu überspringen und trägt somit zur Reduktion von Auslassungsfehlern bei. Zusätzlich kann z. B. bei der Spracheingabe mittels Prüfziffern kontrolliert werden, ob die richtige Menge oder der richtige Artikel entnommen wurden.

Obwohl ein zusätzlicher Schritt der Quittierung bereits dafür sorgen kann, dass der Kommissionierer nochmals kurz reflektiert, ob er tatsächlich den richtigen Artikel oder die richtige Stückzahl entnommen hat, bleibt der Mensch als wesentliche Fehlerursache erhalten. Der Einsatz von Identsystemen kann diesen Nachteil kompensieren, indem eine Kontrolle von Prozessschritten während der Kommissionierung vorgenommen wird. Eine gängige Methode ist der Einsatz von Barcodescannern, mit denen z. B. der Barcode eines Lagerfachs oder Artikels gescannt wird. Auch wenn Barcodescanner oftmals in mobilen Datenterminals eingebaut sind, beschränkt sich ihr Einsatz nicht auf diese Geräte. Prinzipiell können Identsysteme zur Fehlervermeidung mit allen Medien der Informationsbereitstellung kombiniert werden. In Abhängigkeit davon, ob jede einzelne Entnahmeeinheit gescannt werden soll oder z. B. nur der erste Artikel einer Position, wird ein wirkungsvoller Schutz gegen Auslassungs-, Typ- und/ oder Mengenfehler erzielt. Dies setzt jedoch voraus, dass entsprechende Codes vorhanden sind.

Gerade bei der Kommissionierung von Kleinteilen ist diese Voraussetzung meist nicht gegeben. Deshalb muss hier auf andere Identtechniken zurückgegriffen werden. Häufig werden Waagen eingesetzt, die neben einer Erleichterung beim Zählen hoher Stückzahlen auch eine Kontrollfunktion bieten. Die Verfügbarkeit der Artikelgewichte im Warehouse Management System und ein Sortiment mit ausreichenden Gewichtsunterschieden der Artikel vorausgesetzt - ist auch beim Kontrollwiegen die Reduktion von Auslassungs-, Mengen- und Typfehlern möglich.

Eine weitere Möglichkeit zur Reduktion insbesondere von Typfehlern ist die Überprüfung, ob der Kommissionierer in das richtige Lagerfach gegriffen hat. Technisch kann dies z. B. durch Lichtgitter oder Laserscanner realisiert werden, die die Regalfront überwachen. Auch durch den Einsatz der Radio-Frequency Identification Technologie (RFID-Technologie) ist es möglich, den Griff in das richtige Lagerfach zu überwachen. Der Kommissionierer trägt hierzu ein mobiles RFID-Lesegerät in Form eines Handschuhs, das während des Pickvorgangs am Regal angebrachte Transponder ausliest [Gün11].

Der beste Prozess und die besten technischen Hilfsmittel helfen wenig, wenn die Mitarbeiter nicht hinsichtlich der Fehlervermeidung sensibilisiert sind. Zum einen muss dem Mitarbeiter nahe gebracht werden, welche Auswirkungen Kommissionierfehler auf den Erfolg des Unternehmens haben. In der Regel führt die Darstellung der Wichtigkeit der Aufgabe und der damit verbundenen Wertschätzung zu einer Motivationssteigerung bei den Kommissionierern. Eine Leistungsprämie kann für zusätzliche Anreize sorgen. Dabei ist darauf zu achten, dass die Pickleistung nicht im Widerspruch zur Qualität steht. Die Leistung muss sich auch aus der Anzahl der richtigen Picks ergeben.

\section{FEHLERVERMEIDUNG DURCH PICK-BY-VISION}

Neben bisher bereits in der Praxis eingesetzten technischen Hilfsmitteln, wie beispielsweise Pick-by-VoiceSystemen oder Barcodescannern, wird im Bereich der Forschung seit einiger Zeit an dem Augmented-Realitybasierten Kommissioniersystem Pick-by-Vision gearbeitet. 
Die Augmented Reality (AR) umfasst im Allgemeinen die situationsbezogene und perspektivisch richtige Einblendung virtueller Information in das Sichtfeld des Nutzers und die Interaktion in Echtzeit [Azu97]. Bei Pickby-Vision-Systemen werden alle für den Kommissioniervorgang relevanten Daten mittels eines Head-mounted Displays (HMD) zur Verfügung gestellt. Abbildung 3 zeigt den Blick durch das HMD während der Kommissionierung. In der dargestellten Situation werden dem Kommissionierer die Artikelnummer, die zu entnehmende Stückzahl und das Lagerfach eingeblendet. Während die Artikelnummer und die Stückzahl links bzw. rechts oben im HMD in Textform angezeigt werden, erhält der Kommissionierer bei der Wegfindung im Lager visuelle Unterstützung. Bei dem in Abbildung 3 gezeigten System wird ein sich dynamisch anpassender Tunnel für das intuitive Auffinden des richtigen Lagerfachs verwendet [Scw10].

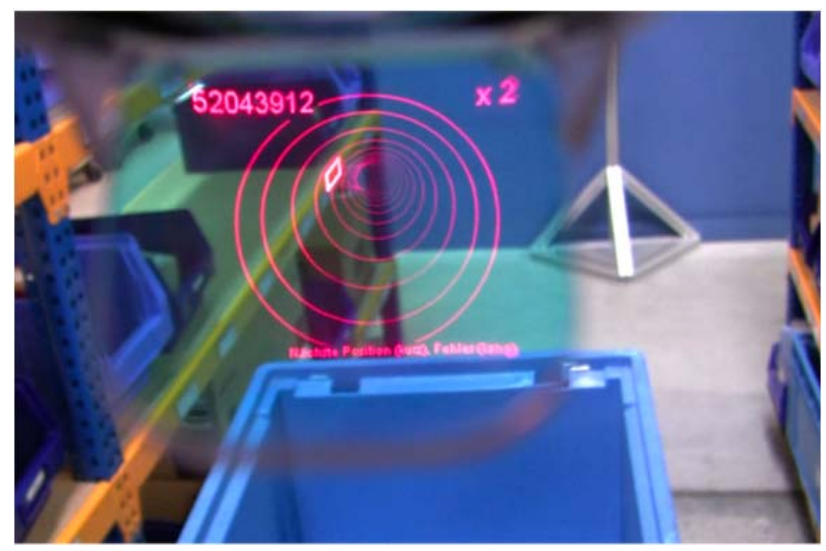

Abbildung 3. Wegfindung im Lager mittels eines virtuellen sich dynamisch anpassenden Tunnels

Dass diese Art der Informationsbereitstellung die schnelle und fehlerfreie Informationsaufnahme unterstützt, bestätigt auch eine am Lehrstuhl fml durchgeführte Probandenstudie. Unter Verwendung eines AR-unterstützten Kommissioniersystems mit visueller Lagernavigation erreichten die Probanden im Mittel eine $10 \%$ höhere Kommissionierleistung als mit der konventionellen $\mathrm{Pa}-$ pierliste, während gleichzeitig die Fehlerrate um durchschnittlich 50 \% niedriger lag [Gün09].

Neben dem Vorteil der intuitiven Informationsbereitstellung kann auch bei Pick-by-Vision die schrittweise Auftragsabarbeitung realisiert werden. Ähnlich wie bei Pick-by-Light- oder Pick-by-Voice-Systemen werden einzelne Prozessschritte so gestaltet, dass für die weitere Auftragsbearbeitung die Quittierung durch den Kommissionierer erforderlich ist. Hierfür kommt als Interaktionsgerät oftmals ein Dreh-Drückknopf am Gürtel oder Spracheingabe zum Einsatz.

Durch die Integration einer Kamera in das HMD und den Einsatz entsprechender Videoauswertungssoftware kann die Interaktion vereinfacht und hinsichtlich der Feh- lervermeidung weiter verbessert werden. Abbildung 4 zeigt den Blick durch das HMD eines solchen Systems.

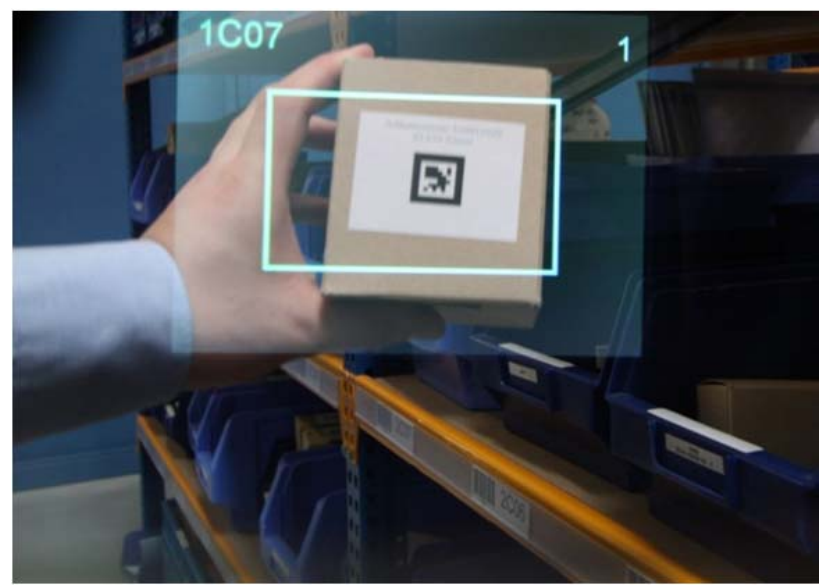

Abbildung 4. Überprüfung optischer Codes mittels einer Kamera im Head-mounted Display

Nach der Entnahme eines Artikels wird dieser kurz so in den Rahmen gehalten, dass die Kamera diesen identifizieren kann. Vergleichbar mit einem Barcode-Scanner wird unmittelbar nach der Entnahme eine optische Codierung auf der Entnahmeeinheit erfasst. Im Gegensatz zum Barcodescanner stehen dem Kommissionierer bei der Arbeit mit dem Pick-by-Vision-System beide Hände für die eigentliche Kommissionieraufgabe zur Verfügung. Darüber hinaus ermöglicht die Auswertung des Kamerabildes eine schnelle und zuverlässige Erfassung, auch wenn der Code nicht exakt gegenüber der Kamera ausgerichtet ist. Somit leistet Pick-by-Vision nicht nur einen Beitrag zur Vermeidung von Fehlern sondern auch zur Erhöhung der Kommissionierleistung.

\section{AUFWAND UND NUTZEN DER FEHLERVERMEIDUNG}

Jeder zusätzliche Vorgang zum Erkennen von Fehlern erfordert Zeit und damit Geld bzw. die Anschaffung zusätzlicher Technik. Durch diese Maßnahmen sinkt das Risiko, dass ein Fehler unentdeckt bleibt. Unentdeckte Fehler verursachen wiederum auch Kosten, die auf Retouren sowie Nachlieferungen und im schlimmsten Fall sogar den Verlust des Kunden und damit Umsatzeinbußen zurückzuführen sind.

Unter den gegebenen Randbedingungen ist ein optimales Verhältnis zwischen den Kosten für die Fehlervermeidung und den Kosten, die durch Fehler entstehen, zu finden. Das Optimum ist dabei nicht genau zu bestimmen, da vor allem die Fehlerkosten nur ungenau spezifiziert werden können. Allgemein kann festgestellt werden, dass die Kosten für die Fehlerbehebung steigen, um so später der Fehler entdeckt wird. Fehler, die also direkt bei der Entnahme erkannt und behoben werden können, verursachen die geringsten bis gar keine Kosten. Fehler, die z. B. erst bei Stichprobenkontrollen im Warenausgang erkannt 
werden, verursachen höhere Kosten. In einer stichprobenartigen Umfrage des Lehrstuhls fml wurden für diesen Fall die Kosten für die Behebung des Fehlers mit 25 Euro beziffert. Wird der Fehler erst vom Kunden selbst entdeckt, so fallen Retouren-, neue Auftragsbearbeitungsund Versandkosten an. In diesem Fall wurden durchschnittlich Kosten von 210 Euro für die Fehlerbehebung von den befragten Unternehmen angegeben.

In Abhängigkeit der unternehmerischen Ziele sind Aufwand und Nutzen der Fehlervermeidung in Einklang zu bringen. Wird z. B. für die Kontrolle der kommissionierten Ware der Prozess dahin gehend verändert, dass jeder Artikel gescannt wird, so erhöht sich der Zeitaufwand pro Entnahmeeinheit laut einer MTM-Zeitstudie um ca. 4 Sekunden. Dies hat zur Folge, dass die Kommissionierkosten pro Entnahmeeinheit um ca. 1,7 Cent steigen. In Abhängigkeit der mit dieser Maßnahme verbundenen Fehlerreduzierung lässt sich der monetäre Nutzen bewerten.

In einem Fallbeispiel sind täglich 500 Aufträge mit durchschnittlich 4 Positionen und 2 Entnahmeeinheiten zu kommissionieren. Wenn jede Entnahmeeinheit zusätzlich gescannt werden soll, sind in Summe 4.000 Scanvorgänge notwendig. Unter Berücksichtigung von Fehllesungen, des Handhabungsaufwands des Scanners und der Korrektur des erkannten Fehlers ergeben sich Fehlervermeidungskosten von 70-100 Euro pro Tag. Eine Reduktion dieses Aufwands kann durch den Einsatz technischer Hilfsmittel erreicht werden, die keinen zusätzlichen Handhabungsaufwand erzeugen. Ein vielversprechender Ansatz hierfür ist das Erkennen der gegriffenen Entnahmeeinheit durch eine Kamera, die der Kommissionierer am Körper trägt, um dessen Arbeitsbereich zu überwachen. Ein solches System wurde im Rahmen des Forschungsprojektes „Pick-by-Vison II“ erprobt. Bisher sind solche Systeme für den Praxiseinsatz noch nicht nutzbar.

Abschließend lässt sich festhalten, dass für die Auswahl der richtigen Fehlervermeidungsmaßnahmen sowohl die Rahmenbedingungen als auch die Kosten, die durch Fehler verursacht werden, entscheidend sind. Nicht für jeden Anwendungsfall ist eine $100 \%$-Kontrolle und damit das Ziel einer „Null-Fehler-Kommissionierung“ erstrebenswert. Unabhängig von der Prozessgestaltung und dem Einsatz technischer Hilfsmittel ist es wichtig, den Mitarbeitern die Auswirkungen von Kommissionierfehlern zu verdeutlichen und damit die Motivation und Achtsamkeit der Kommissionierer zu erhöhen.

\section{$7 \quad$ FAZIT}

Auch wenn eine Fehlerquote von 0,3 \% auf den ersten Blick sehr nierig erscheint, so bedeutet dies dennoch, dass etwa jede 300. kommissionierte Position fehlerbehaftet ist. Je nachdem, ob und wo der Fehler entdeckt wird, sind die Folgen unterschiedlich zu bewerten. Je früher ein Fehler entdeckt wird, desto einfacher kann dieser korri- giert werden. Fehler, die erst beim Kunden entdeckt werden, können schwerwiegende Konsequenzen haben. Dies beginnt bei Aufwendungen für Retouren und Expresslieferungen und kann bis zu finanziellen Entschädigungen für einen Produktionsstillstand führen. Um den Unternehmenserfolg nicht zu gefährden, wird häufig die Fehlerkontrolle an der Schnittstelle zum Kunden, dem Warenausgang, durchgeführt.

Für eine systematische Reduzierung der Fehlerquellen ist eine Untersuchung der Ursachen für Kommissionierfehler erforderlich. Den identifizierten Ursachen kann durch gezielte Maßnahmen entgegengewirkt werden. Ein ganzheitlicher Ansatz zur Auswahl geeigneter Maßnahmen muss sowohl die Technik, den Prozess, die Organisation und natürlich auch die Motivation der Mitarbeiter beachten.

Der Aufwand für die angewandten Maßnahmen zur Fehlerreduzierung muss in einem vernünftigen Verhältnis zu dem zu erwartenden Nutzen, respektive der zu erreichenden Reduzierung der Fehlerquote, stehen. Technologien, die eine Fehlererkennung unterstützen und dabei keinen zusätzlichen Handhabungsaufwand hervorrufen, können ihren Platz im Spannungsfeld zwischen Aufwand und Nutzen finden. Ein vielversprechender Ansatz für die Zukunft ist das am Lehrstuhl fml entwickelte Konzept auf Basis der Pick-by-Vision-Technologie. In der Umsetzung am Lehrstuhl fml prüft ein Bilderkennungssystem, ob der Mitarbeiter den richtigen Artikel entnommen hat. Dies erfolgt im Hintergrund gleichzeitig zum Greifvorgang und beansprucht somit keine zusätzliche Zeit. Solche technologischen Ansätze können künftig in klassischen Personzur-Ware-Systemen die Fehlerquote deutlich reduzieren. 


\section{LITERATUR}

[Arn03] Arnold, Dieter: Materialfluss in Logistiksystemen. Berlin: Springer, 2003

[Azu97] Azuma, Ronald T.: A survey of Augmented Reality. In: Presence: Teleoperators and Virtual Environments. Hughes Research Laboratories, Malibu, 1997

[Bau08] Bauer, Nikolas: Die Fabrik von heute für das Auto von morgen. In: Baumgarten, Helmut (Hrsg.): Das Beste der Logistik: Innovationen, Strategien, Umsetzungen. Berlin, Heidelberg: Springer Verlag, 2008

[Dul05] Dullinger, Karl-Heinz: Das richtige Kommissionier-Konzept - eine klare Notwendigkeit, In: Jahrbuch Logistik 2005. Düsseldorf: Verlagsgruppe Handelsblatt, 2005

[Fra01] Frazelle, Edward: Word-Class Warehousing and Material Handling. New York: McGraw-Hill, 2001

[Gün09] Günthner, Willibald A.; Blomeyer, Niels; Reif, Rupert; Schedlbauer, Michael: Pick-by-Vision: Augmented Reality unterstützte Kommissionierung. TU München, Abschlussbericht, München, 2009

[Gün11] Günthner, Willibald A.; Wölfle, Michael: Papierlose Produktion und Logistik.
TU München, Forschungsbericht, München, 2011

[Lol03] Lolling, Andreas: Analyse der menschlichen Zuverlässigkeit bei Kommissioniertätigkeiten. Dissertation, Aachen: Shaker Verlag, 2003

[Pia11] Piazza, Hans-Martin: Automatisierte Pick-Lösungen im Kommen. In: FM Das Logistik-Magazin 3/2011, Stuttgart: Verlag Industrie und Logistik, 2011

[Scw10] Schwerdtfeger, Björn: Pick-by-Vision: Bringing HMD-based Augmented Reality into the Warehouse. TU München, Dissertation, München, 2010

[Str05] Straube, Frank; Pfohl, Hans-Christian; Günthner, Willibald A.; Dangelmaier Wilhelm: Trends und Strategien in der Logistik: Ein Blick auf die Agenda des Logistik-Managements 2010. Hamburg: Deutscher Verkehrs-Verlag, 2005

[Str08] Straube, Frank; Pfohl, Hans-Christian: Trends und Strategien in der Logistik 2008: Globale Netzwerke im Wandel. Bremen: DVV, 2008

[Wei04] Weineck, Jürgen: Sportbiologie. Balingen: Spitta-Verlag, 2004
Das IGF-Vorhaben 16398 N/1 der Forschungsvereinigung Bundesvereinigung Logistik e.V. - BVL, Schlachte 31, 28195 Bremen wurde über die AiF im Rahmen des Programms zur Förderung der industriellen Gemeinschaftsforschung und -entwicklung (IGF) vom Bundesministerium für Wirtschaft und Technologie aufgrund eines Beschlusses des Deutschen Bundestages gefördert.
Gefördert durch:

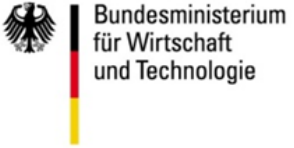

aufgrund eines Beschlusses des Deutschen Bundestages 